\title{
EFFICIENT SAMPLING OF SPARSE WIDEBAND ANALOG SIGNALS
}

\author{
Moshe Mishali and Yonina C. Eldar \\ Department of Electrical Engineering \\ Technion - Israel Institute of Technology \\ Haifa, Israel 32000 \\ \{moshiko@tx,yonina@ee\}.technion.ac.il
}

\author{
Joel A. Tropp \\ Applied \& Computational Mathematics \\ California Institute of Technology \\ Pasadena, CA 91125-5000 \\ jtropp@acm.caltech.edu
}

\begin{abstract}
Periodic nonuniform sampling is a known method to sample spectrally sparse signals below the Nyquist rate. This strategy relies on the implicit assumption that the individual samplers are exposed to the entire frequency range. This assumption becomes impractical for wideband sparse signals. The current paper proposes an alternative sampling stage that does not require a full-band front end. Instead, signals are captured with an analog front end that consists of a bank of multipliers and lowpass filters whose cutoff is much lower than the Nyquist rate. The problem of recovering the original signal from the low-rate samples can be studied within the framework of compressive sampling. An appropriate parameter selection ensures that the samples uniquely determine the analog input. Moreover, the analog input can be stably reconstructed with digital algorithms. Numerical experiments support the theoretical analysis.
\end{abstract}

Index Terms - Analog to digital conversion, compressive sampling, infinite measurement vectors (IMV), multiband sampling.

\section{INTRODUCTION}

Radio frequency (RF) technology enables the modulation of a narrowband signal by a high carrier frequency. As a consequence, manmade radio signals are often sparse. That is, they consist of relatively small number of narrowband transmissions spread across a wide territory of spectrum. A convenient description for these signals is the multiband model where the frequency support of a signal resides within several continuous intervals in a wide spectrum but vanishes elsewhere.

It has become prohibitive to sample modern multiband signals because their Nyquist rates may exceed the specifications of the best analog-to-digital converters (ADCs) by orders of magnitude. As a result, any attempt to acquire a multiband signal must exploit its structure in an intelligent way.

Previous work on multiband signals has shown that it is possible to reduce the sampling rate by acquiring samples from a periodic but nonuniform grid [1]. Multi-coset sampling, a specific strategy of this type, was analyzed in [2], which established that exact recovery is possible when the band locations are known. The blind case, in which the band locations are unknown, has been extensively studied in [3]. Unfortunately, the sampling front ends proposed in [1-3] are impractical for wideband applications because they require ADCs

This work was supported in part by the Israel Science Foundation under Grant no. 1081/07 and by the European Commission in the framework of the FP7 Network of Excellence in Wireless COMmunications NEWCOM++ (contract no. 216715).

JAT was supported in part by DARPA and ONR. whose sampling rate is matched to the Nyquist rate of the input signal, even when the average sampling rate is much lower. Other limitations are described in Section 2.2. Another recent work [4] has partially overcome these shortcomings using a hybrid optic-electronic system at the expense of size and cost.

In this paper, we analyze a practical sampling system inspired by the recent work on the random demodulator [5]. This system multiplies the input signal by a random square wave alternating at the Nyquist rate, then it performs lowpass filtering, and samples the signal at a lower rate. Our system consists of a bank of random demodulators running in parallel. We show that, for an appropriate choice of parameters, our system uniquely and stably determines a multiband input signal. Moreover, we describe digital algorithms for reconstructing the signals from the parallel samples.

We continue with an outline of the paper. Section 2 reviews essential background material. In Section 3, we describe the system design and a frequency-domain analysis that leads to an infinite measurement vectors (IMV) system. Applying ideas from [6], we reduce the problem of locating the frequency bands to a finitedimensional compressive sampling problem. We then derive an appropriate choice of parameters for the sampling system. Section 4 presents our numerical experiments, which demonstrate that the system permits stable signal recovery in the presence of noise.

\section{FORMULATION AND BACKGROUND}

\subsection{Design Goals for Efficient Sampling}

Let $x(t)$ be a real-valued, finite-energy, continuous-time signal, and let $X(f)=\int_{-\infty}^{\infty} x(t) \exp (-j 2 \pi f t) \mathrm{d} t$ be its Fourier transform. We treat a multiband signal model $\mathcal{M}$ in which $x(t)$ is bandlimited to $\mathcal{F}=\left[-f_{\mathrm{NYQ}} / 2, f_{\mathrm{NYQ}} / 2\right]$ and the support of $X(f)$ consists of at most $2 N$ frequency interval whose widths do not exceed $B$. Fig. 1 depicts a typical communication application that obeys this signal model.

We wish to design a sampling system for signals from our model $\mathcal{M}$ that satisfies the following properties:

1. The sampling rate should be as low as possible;

2. the system has no prior knowledge of band locations; and

3. the system can be implemented with existing devices.

We will call this type of sampling stage efficient.

The set $\mathcal{M}$ is a union of subspaces corresponding to all possible signal supports. Every $x(t) \in \mathcal{M}$ lies in one of these subspaces. Detecting the exact subspace, prior to sampling, may be impossible or too expensive to implement. An efficient system should therefore be blind, in the sense that band locations are not assumed to be known. 


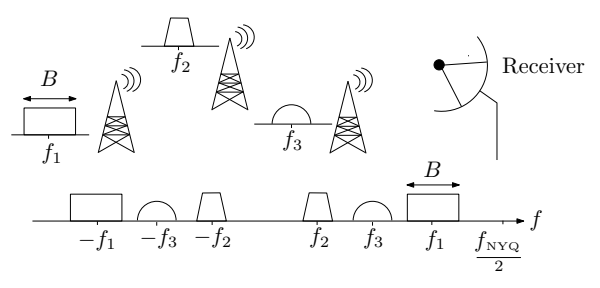

Fig. 1: Three RF transmissions with different carriers $f_{i}$. The receiver sees a multiband signal (bottom drawing). In this example $N=3$, and the modulation techniques of the transmitters determine the maximal expected width $B$.

The lowest (average) sampling rate that allows blind perfect reconstruction for all signals in $\mathcal{M}$ is $4 N B$ samples/sec [3]. This rate is proportional to the effective bandwidth of $x(t)$, and it is typically far less than the Nyquist rate $f_{\mathrm{NYQ}}$, which depends only on the maximum frequency in $x(t)$. See Section 3.3 for more discussion.

Our previous work describes blind reconstruction of $x(t) \in \mathcal{M}$ from multi-coset samples taken at the minimal rate [3]. The next section details the practical limitations of the multi-coset strategy, which make it inefficient for wideband signals.

\subsection{Practical Limitations of Multi-Coset Sampling}

Multi-coset sampling involves periodic nonuniform sampling of the Nyquist-rate sequence $x\left(n T_{\mathrm{NYQ}}\right)$, where $T_{\mathrm{NYQ}}=1 / f_{\mathrm{NYQ}}$. The $i$ th coset takes the $i$ th value in every block of $L$ consecutive samples. Retaining only $p<L$ cosets, indexed by $C=\left\{c_{i}\right\}_{i=1}^{p}$, gives $p$ sequences

$$
x_{c_{i}}[n]= \begin{cases}x\left(n T_{\mathrm{NYQ}}\right) & n=m L+c_{i}, m \in \mathbb{Z} \\ 0 & \text { otherwise, }\end{cases}
$$

with an average sampling rate $p /\left(L T_{\mathrm{NYQ}}\right)$, which is lower than the Nyquist rate.

To explain the practical limitations of this strategy, we observe that standard ADC devices have a specified maximal rate $r$, and manufactures require a preceding low-pass filter with cutoff $r / 2$. Distortions occur if the anti-aliasing filter is not used, since the design is tailored to $r / 2$-bandlimited signals and has an internal parasitic response to frequencies above $r / 2$. To avoid these distortions, an ADC with $r$ matching the Nyquist rate of the input signal must be used, even if the actual sampling rate is below the maximal conversion rate $r$. In multi-coset sampling, each sequence $x_{c_{i}}[n]$ corresponds to uniform sampling at rate $1 /\left(L T_{\mathrm{NYQ}}\right)$, whereas the input $x(t)$ contains frequencies up to $f_{\mathrm{NYQ}} / 2$. Acquiring $x_{c_{i}}[n]$ is only possible using an ADC with $r=f_{\mathrm{NYQ}}$, which runs $L$ times slower than its maximal rate. Besides the resource waste, this renders multi-coset sampling impractical in wideband applications where $f_{\mathrm{NYQ}}$ is higher (typically by orders of magnitude) than the rate $r$ of available devices.

One recent paper [4] developed a nonconventional ADC design for wideband applications by means of high-rate optical devices. The hybrid optic-electronic system allows sampling at rate $1 /\left(L T_{\mathrm{NYQ}}\right)$ with minimal attenuation to higher frequencies (up to $\left.f_{\mathrm{NYQ}} / 2\right)$. Unfortunately, at present, this performance cannot be achieved with purely electronic technology. Thus, for wideband applications that cannot afford the size or expense of an optical system, multi-coset sampling becomes impractical.

Another limitation of multi-coset sampling, which also exists in the optical implementation, is maintaining accurate time delays between the ADCs of different cosets. Any uncertainty in these delays hobbles the recovery from the sampled sequences.

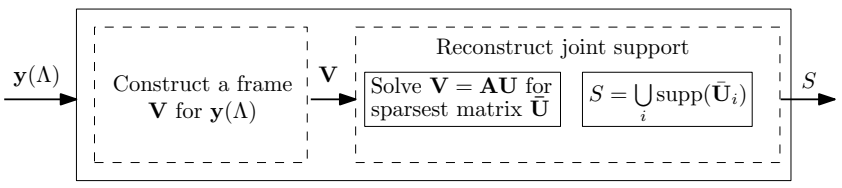

Fig. 2: Recovery of the joint support $S=\operatorname{supp}(\mathbf{x}(\Lambda))$.

Before describing the way our proposed sampling stage overcomes these limitations, we briefly review the mechanism underlying the blind reconstruction of [3].

\subsection{IMV System}

Let $\mathbf{x}(\Lambda)=\{\mathbf{x}(\lambda): \lambda \in \Lambda\}$ be a collection of $n$-dimensional vectors indexed by a fixed set $\Lambda$ that may be infinite. The support of a vector is the $\operatorname{set} \operatorname{supp}(\mathbf{v})=\left\{i \mid \mathbf{v}_{i} \neq 0\right\}$, and we define $\operatorname{supp}(\mathbf{x}(\Lambda))=\cup_{\lambda} \operatorname{supp}(\mathbf{x}(\lambda))$. We will assume that the vectors in $\mathbf{x}(\Lambda)$ are jointly $K$-sparse in the sense that $|\operatorname{supp}(\mathbf{x}(\Lambda))| \leq K$. In words, the nonzero entries of each vector $\mathbf{x}(\lambda)$ lie within a set of at most $K$ indices.

Let $\mathbf{A}$ be an $m \times n$ matrix with $m<n$, and consider a parameterized family of linear systems

$$
\mathbf{y}(\lambda)=\mathbf{A x}(\lambda), \quad \lambda \in \Lambda .
$$

When the support $S=\operatorname{supp}(\mathbf{x}(\Lambda))$ is known, recovering $\mathbf{x}(\Lambda)$ from the known vector set $\mathbf{y}(\Lambda)=\{\mathbf{y}(\lambda): \lambda \in \Lambda\}$ is possible if the submatrix $\mathbf{A}_{S}$, consisting of the columns of $\mathbf{A}$ indicated by $S$, has full column rank. In this case,

$$
\begin{aligned}
\mathbf{x}_{S}(\lambda) & =\left(\mathbf{A}_{S}\right)^{\dagger} \mathbf{y}(\lambda) \\
\mathbf{x}_{i}(\lambda) & =0, \quad i \notin S
\end{aligned}
$$

where $\mathbf{x}_{S}(\lambda)$ contains only the entries of $\mathbf{x}$ indexed by $S$, where $\mathbf{A}_{S}^{H}$ denotes the conjugate transpose of $\mathbf{A}_{S}$ and where $\left(\mathbf{A}_{S}\right)^{\dagger}=$ $\left(\mathbf{A}_{S}^{H} \mathbf{A}_{S}\right)^{-1} \mathbf{A}_{S}^{H}$ is the Moore-Penrose pseudoinverse. For unknown support $S$, (2) is still invertible if $K=|S|$ is known, and every set of $2 K$ columns from $\mathbf{A}$ is linearly independent [6-8]. In general, solving (2) for $\mathbf{x}(\Lambda)$ is NP-hard because it may require a combinatorial search. Nevertheless, recent advances in compressive sampling and sparse approximation delineate situations where polynomial-time recovery algorithms correctly identify $\operatorname{supp}(\mathbf{x}(\Lambda))$ for finite $\Lambda$. This challenge is sometimes referred to as a multiple measurement vectors (MMV) problem [8-13].

Recovering a multiband signal $x(t)$ from a set of multi-coset samples can be reduced to a certain infinite measurement vectors (IMV) problem (where $\Lambda$ is infinite). When the band locations are known, the support set $S$ is determined and reconstruction can be performed via $(3)[1,2]$. In a blind scenario, the support of the unknown vectors $\mathbf{x}(\lambda)$ can be recovered in two steps $[3,6]$. First, construct a (finite) frame $\mathbf{V}$ for $\mathbf{y}(\Lambda)$. Then, find the (unique) solution $\overline{\mathbf{U}}$ to the MMV system $\mathbf{V}=\mathbf{A} \mathbf{U}$ that has the fewest nonzero rows. It holds that $\operatorname{supp}(\mathbf{x}(\Lambda))$ is the set $S=\cup_{i} \operatorname{supp}\left(\overline{\mathbf{U}}_{i}\right)$, where the union occurs over columns of $\overline{\mathbf{U}}$. Fig. 2 summarizes these recovery steps.

In the next section, we describe and analyze the proposed sampling system. In contrast to the multi-coset strategy, our system uses standard low-rate ADCs. We match the analog input of the ADCs to their maximal rate. The system also avoids time offsets between devices. As in multi-coset sampling, the sampling sequences generated by our system are related to $x(t)$ via an IMV system, different 

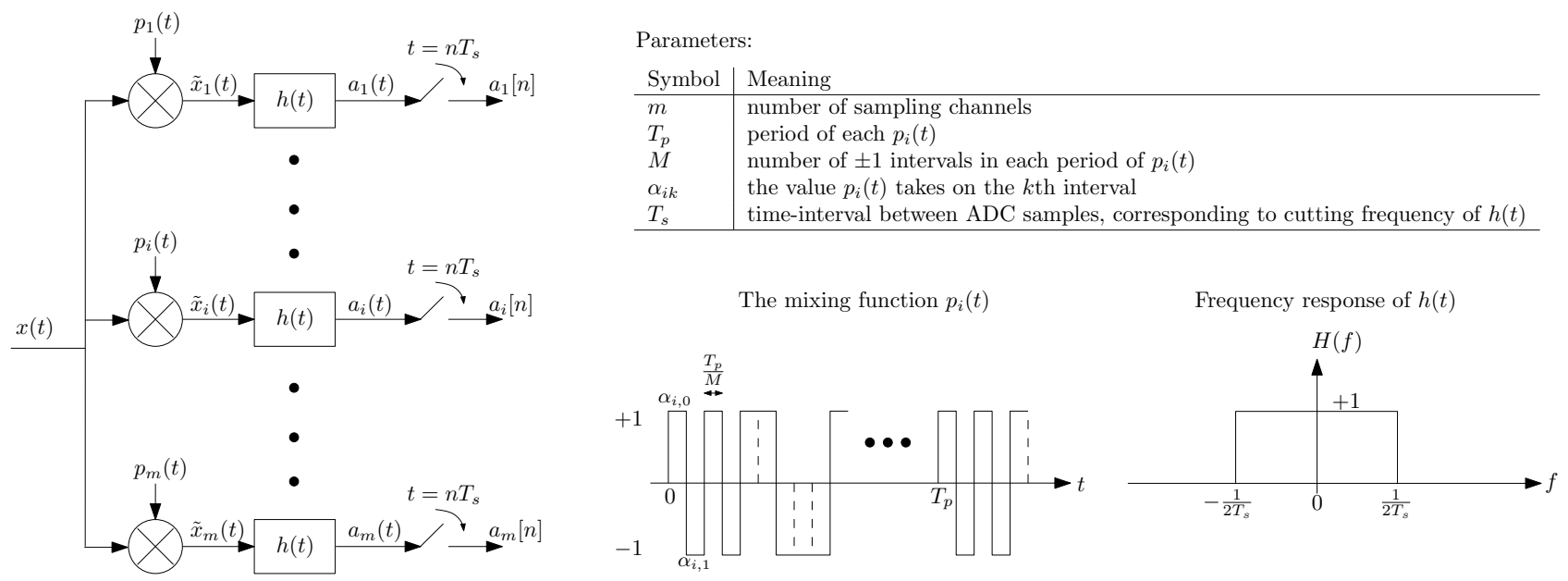

Fig. 3: Description of a practical sampling stage for multiband signals.

from the one which is based on the sequences (1). Consequently, the recovery of $x(t)$ can be performed via the steps described in Fig. 2 and (3).

\section{EFFICIENT SAMPLING}

\subsection{Description}

Let us present the proposed system in more detail. A block diagram appears in Fig. 3. We discuss the choice of system parameters in the sequel.

The signal $x(t)$ enters $m$ channels simultaneously. In the $i$ th channel, $x(t)$ is multiplied by a mixing function $p_{i}(t)$, which is a $T_{p}$-periodic, piecewise constant function that alternates between the levels \pm 1 for each of $M$ equal time intervals. Formally,

$$
p_{i}(t)=\alpha_{i k}, \quad k \frac{T_{p}}{M} \leq t \leq(k+1) \frac{T_{p}}{M}, \quad 0 \leq k \leq M-1,
$$

with $\alpha_{i k} \in\{+1,-1\}$, and $p_{i}\left(t+n T_{p}\right)=p_{i}(t)$ for every $n \in \mathbb{Z}$.

After mixing, the output is converted to digital using the standard approach. In each channel, the signal spectrum is truncated by a lowpass filter with cutoff $1 /\left(2 T_{s}\right)$ and the filtered signal is sampled at rate $1 / T_{s}$

Note that the cutoff and the sampling rate match, and each channel operates independently. Since there are $m$ channels, the average sampling rate is $m / T_{s}$ samples/sec. A further advantage of this type of system is that samples are produced at a constant rate, so they may be fed to a digital processor operating at the same frequency, whereas multi-coset sampling requires an additional hardware buffer to synchronize the nonuniform sequences.

\subsection{Analysis}

To ease exposition we choose an odd $M, T=M / f_{\mathrm{NYQ}}$, and $T_{s}=$ $T_{p}=T$. These choices are relaxed in [14]. Consider the $i$ th channel. Since $p_{i}(t)$ is periodic, it has a Fourier expansion

$$
p_{i}(t)=\sum_{n=-\infty}^{\infty} c_{i n} e^{j \frac{2 \pi}{T} n t},
$$

where the coefficients are given by [14]

$$
c_{i n}=\frac{1}{2 \pi}\left(\sum_{k=0}^{M-1} \alpha_{i k} e^{-j \omega_{0} n k}\right) \frac{1-e^{-j \omega_{0} n}}{j n},
$$

for $\omega_{0}=2 \pi / M$ and $c_{i n}=c_{i,-n}$. Expressing the Fourier transform $P_{i}(f)$ in terms of the Fourier series coefficients $c_{i n}$ leads to

$$
P_{i}(f)=\int_{-\infty}^{\infty} p_{i}(t) e^{-j 2 \pi f t} d t=\sum_{n=-\infty}^{\infty} c_{i n} \delta\left(f-\frac{n}{T}\right),
$$

with $\delta(t)$ denoting the Dirac delta function. The analog multiplication $\tilde{x}_{i}(t)=x(t) p_{i}(t)$ translates to convolution in the frequency domain,

$$
\tilde{X}_{i}(f)=X(f) * P_{i}(f)=\sum_{n=-\infty}^{\infty} c_{i n} X\left(f-\frac{n}{T}\right) .
$$

Therefore, $\tilde{X}_{i}(f)$ is a linear combination of shifted copies of $X(f)$.

Filtering $\tilde{X}_{i}(f)$ by $H(f)$, whose frequency response is an ideal rect function in the interval $\mathcal{F}_{0}=[-1 /(2 T), 1 /(2 T)]$, results in

$$
A_{i}(f)=H(f) \tilde{X}_{i}(f)=\sum_{n=-n_{0}}^{n_{0}} c_{i n} X\left(f-\frac{n}{T}\right), \quad f \in \mathcal{F}_{0},
$$

where $n_{0}$ is the smallest integer satisfying $2 n_{0}+1 \geq T f_{\mathrm{NYQ}}$. Under the choices above, $n_{0}=(M-1) / 2$. The discrete-time Fourier transform of $a_{i}[n]$ is

$$
\begin{aligned}
A_{i}\left(e^{j 2 \pi f T}\right) & =\sum_{n=-\infty}^{\infty} a_{i}[n] e^{-j 2 \pi f T n} \\
& =\sum_{n=-n_{0}}^{n_{0}} c_{i n} X\left(f-\frac{n}{T}\right), \quad f \in \mathcal{F}_{0} .
\end{aligned}
$$

Substituting (6) in (11) leads to the system

$$
\mathbf{y}(f)=(\mathbf{S F})(\mathbf{D} \mathbf{x}(f)), \quad f \in \mathcal{F}_{0},
$$

where $\mathbf{y}_{i}(f)=A_{i}\left(e^{j 2 \pi f T}\right), 1 \leq i \leq m, \mathbf{S}$ is an $m \times M$ matrix whose $i k$ th entry $\mathbf{S}_{i k}=\alpha_{i k}$. The $M \times M$ matrix $\mathbf{F}$ is a certain cyclic 


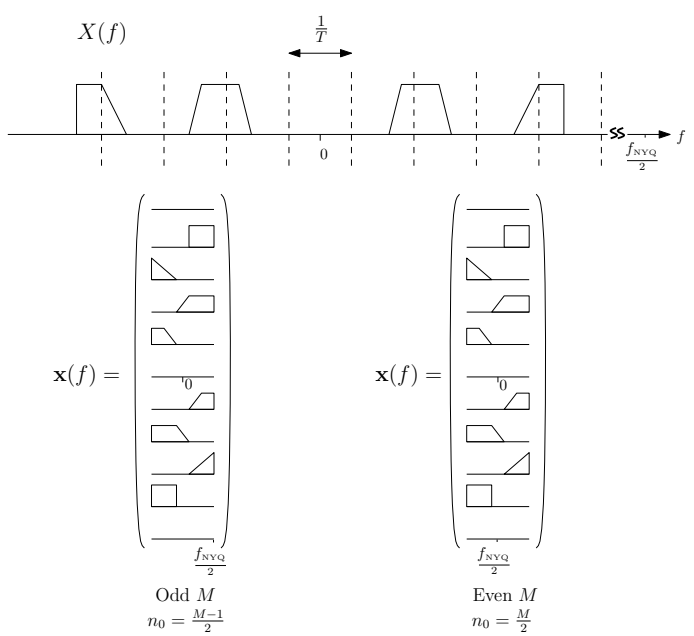

Fig. 4: The relation between the Fourier transform $X(f)$ and the unknown vector set $\mathbf{x}(f)$, eq. (13).

columns shift of the discrete Fourier transform matrix of order $M$. The $M$-square diagonal $\mathbf{D}$ scales

$$
\mathbf{x}_{i}(f)=X\left(f+\left(i-n_{0}-1\right) / T\right)
$$

according to the last term in (6). Since $\mathbf{D}$ has non-zero diagonal entries, it can be absorbed into $\mathbf{x}(f)$ while keeping $\operatorname{supp}\left(\mathbf{x}\left(\mathcal{F}_{0}\right)\right)=$ $\operatorname{supp}\left(\mathbf{D x}\left(\mathcal{F}_{0}\right)\right)$. Thus, (12) is an IMV system with $\mathbf{S F}$ replacing $\mathbf{A}$ of (2).

\subsection{Parameter Selection and Stable Recovery}

The following theorem suggests a parameter selection for which the infinite sequences $a_{i}[n], 1 \leq i \leq m$ match a unique $x(t) \in \mathcal{M}$. When the band locations are known, the same selection works with half as many sampling channels. Thus, the system appearing in Fig. 3 can also replace the multi-coset stage of [2].

Theorem 1 (Uniqueness) Let $x(t) \in \mathcal{M}$ be a multiband signal and assume the choices $T=M / f_{N Y Q}$ for an integer $M$ (not necessarily odd) and $T_{p}=T_{s}=T$. If

1. $M \leq f_{N Y Q} / B$,

2. $m \geq 2 N$ for non-blind reconstruction or $m \geq 4 N$ for blind,

3. $\mathbf{S}=\left\{\alpha_{i k}\right\}$ is such that every $4 N$ columns are linearly independent,

then, for every $f \in \mathcal{F}_{0}$, the vector $\mathbf{x}(f)$ is the unique $2 N$-sparse solution of (12). In addition, under these choices $\mathbf{x}\left(\mathcal{F}_{0}\right)$ is jointly $4 N$-sparse.

Proof. The proof goes along the line of [3]. The relation (13) can be thought of slicing the spectrum $X(f)$ into pieces of length $1 / T$ and then rearranging them in a vector form $\mathbf{x}(f)$. Fig. 4 visualizes this relation for even and odd $M$.

The choice $M \leq f_{\mathrm{NYQ}} / B$ ensures that $1 / T \geq B$ and thus every band can contribute only a single non-zero value to $\mathbf{x}(f)$. As a consequence, $\mathbf{x}(f)$ is $2 N$-sparse for every $f \in \mathcal{F}_{0}$. In addition, this choice of $M$ and the continuity of the bands guarantee that each band can occupy two spectrum pieces at the most. Therefore, when aggregating the frequencies to compute $S=\operatorname{supp}\left(\mathbf{x}\left(\mathcal{F}_{0}\right)\right)$, we have $|S| \leq 4 N$.
In the non-blind setting, the band locations imply the support set $S$. The other two conditions on $m, \mathbf{S}$ ensure the existence of $\left(\mathbf{A}_{S}\right)^{\dagger}$, and thus (3) provides the uniqueness of $\mathbf{x}(f)$.

In blind recovery, $S$ is unknown, and the following CS result is used to ensure the uniqueness. A $K$-sparse vector $\mathbf{x}$ is the unique solution of $\mathbf{y}=\mathbf{A x}$ if every $2 K$ columns of $\mathbf{A}$ are linearly independent [7]. Clearly, this condition translates to $m \geq 4 N$ and the condition on $\mathbf{S}$ of the theorem.

The parameter selection of Theorem 1 guarantees an average sampling rate $m / T \geq 4 N B$. Depending on whether $f_{\mathrm{NYQ}} / B$ is an integer, this selection allows to achieve the minimal rate when taking the extreme values for $m, M$. Note that each $\mathbf{x}(f)$ is $2 N$-sparse, while $\mathbf{x}\left(\mathcal{F}_{0}\right)$ is jointly $4 N$-sparse under the parameter selection of the theorem. As detailed in [3], this factor requires doubling $m$ in order to use Fig. 2 and (3). Gaining back this factor at the expense of a higher recovery complexity is also described in [3].

Verifying that a set of signs $\left\{\alpha_{i k}\right\}$ satisfies the requirement of the theorem is computationally difficult because one must check the rank of every set of $4 N$ columns from $\mathbf{S}$. It is known that a random choice of signs will work, except with probability exponentially small in $M$ [15].

In fact, recent work on compressive sampling shows that a random choice of signs ensures that signal acquisition is stable [9]. A matrix $\mathbf{A}$ is said to have the restricted isometry property (RIP) of order $K$, if there exists $0 \leq \delta_{K}<1$ such that

$$
\left(1-\delta_{K}\right)\|\mathbf{x}\|^{2} \leq\|\mathbf{A} \mathbf{x}\|^{2} \leq\left(1+\delta_{K}\right)\|\mathbf{x}\|^{2}
$$

for every $K$-sparse vector $\mathbf{x}$ [9]. When $\mathbf{A}=\mathbf{S F}$ satisfies the RIP of order $4 N$, then the matrices $\mathbf{A}_{S}$ and $\left(\mathbf{A}_{S}\right)^{\dagger}$ are well conditioned for every possible frequency subset $S \subseteq \mathcal{F}_{0}$ with $|S| \leq 2 N$. This fact implies stable recovery, in the sense that the reconstruction error is controlled by the error in the samples [14].

It remains to quantify when stable recovery is possible for specific choices of $m$ and $M$. Let $\mathbf{F}$ be an $M \times M$ unitary matrix (such as $\mathbf{F}$ in (12)), and suppose that $\mathbf{S}$ is an $m \times M$ random matrix whose entries are equally likely to be $\pm 1 / \sqrt{m}$. The RIP of order $K$ holds with high probability for the matrix $\mathbf{A}=\mathbf{S F}$ when $m \geq C K \log (M / K)$, where $C$ is a positive constant independent of everything [16]. The log factor is necessary [17]. In practice, we empirically evaluate the stability of the system since the RIP cannot be verified computationally.

\section{NUMERICAL EVALUATION}

To evaluate the empirical performance of the proposed system (see Fig. 3), we can simulate the action of the system on test signals contaminated with white Gaussian noise. To recover the signals from the sequences of samples, we apply the reduction from an IMV system to an MMV system, as described in Fig. 2. We solve the resulting MMV systems using simultaneous orthogonal matching pursuit $[11,12]$.

More precisely, we evaluate the performance on 100 noisy test signals of the form $x(t)+w(t)$, where $x$ is a multiband signal and $w$ is a white Gaussian noise process. The multiband signals consist of $N=3$ pairs of bands, each of width $B=40 \mathrm{MHz}$, constructed using the formula

$$
x(t)=\sum_{i=1}^{N} \sqrt{E_{i} B} \operatorname{sinc}(B t) \cos \left(2 \pi f_{i} t\right),
$$

where $\operatorname{sinc}(x)=\sin (\pi x) /(\pi x)$. The energy coefficients are fixed $E_{i}=\{1,2,3\}$, whereas for every signal the carriers $f_{i}$ are cho- 


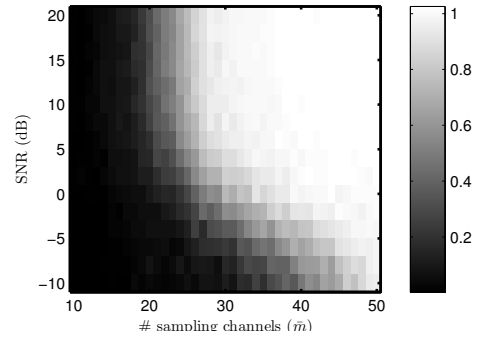

Fig. 5: Image intensity represents percentage of correct support set recovery $\hat{S}=S$, for reconstruction from different number of sampling sequences $\bar{m}$ and under several SNR levels.

sen uniformly at random in $\left[-f_{\mathrm{NYQ}} / 2, f_{\mathrm{NYQ}} / 2\right]$ with $f_{\mathrm{NYQ}}=$ $10 \mathrm{GHz}$. To represent the continuous signals in simulation, we place a dense grid of 4000 equispaced points in the time interval $\left[-200 / f_{\mathrm{NYQ}}, 200 / f_{\mathrm{NYQ}}\right]$. The Gaussian noise is added and scaled so that the test signal has the desired signal-to-noise ratio (SNR), where we define the SNR to be $10 \log (\|x\| /\|w\|)$.

We simulate the proposed system with $m=51$ channels, where each mixing function $p_{i}(t)$ alternated sign at most $M=51$ times. The sampling rate parameters are chosen so that $T_{s}=T_{p}=$ $M / f_{\mathrm{NYQ}}$. Each sign $\alpha_{i k}$ is chosen uniformly at random and fixed for the duration of the experiment. To simulate the analog lowpass filter, we use a 50-tap digital FIR filter, designed with the MATLAB command $h=f i r 1(50,1 / M)$. The output of the filter is decimated to produce the sampled sequences $a_{i}[n]$.

The input signal is reconstructed from $\bar{m} \leq m$ channels. We follow the procedure described in Fig. 2 to obtain an estimated support set $\hat{S}$. When $\hat{S}=S$, the true support set, we declare that the system has recovered the signal. Fig. 5 reports the percentage of recoveries for various numbers $\bar{m}$ of channels and various SNRs.

To construct the frame $\mathbf{V}$, we begin by computing the $m^{2}$ values $\mathbf{Q}_{i k}=\sum_{n} a_{i}[n] a_{k}[n]$. We then perform the eigenvalue decomposition $\mathbf{Q}=\mathbf{V} \mathbf{V}^{H}$ and then discard the eigenvectors of the noise space [3].

\section{CONCLUSIONS}

We developed an efficient sampling stage for analog multiband signals. In the proposed system, analog mixers and standard ADCs replace impractical nonuniform sampling of multi-coset strategy. Analog mixers for wideband applications is an existing RF technology, though selecting the exact devices may require an expertise in analog design.

The proposed system has a set of parameters, which determines the signal, if selected according to the conditions we derived. Analyzing our system in the frequency domain lead to an IMV system, which allows to use existing reconstruction stages with only minor modifications. In addition, based on the IMV system and recent works in the CS literature, we deduce the rate requirements for stable blind recovery, which in general is higher than the rate required to determine the signal from its samples.

A preliminary computer evaluation of our system shows a promise for stable blind recovery from sub-Nyquist sampling rate, although further work is required to quantify the optimal working point in the trade-off between sampling rate, blindness, and practical implementation.

\section{REFERENCES}

[1] Y.-P. Lin and P. P. Vaidyanathan, "Periodically nonuniform sampling of bandpass signals," IEEE Trans. Circuits Syst. II, vol. 45, no. 3, pp. 340-351, Mar. 1998.

[2] R. Venkataramani and Y. Bresler, "Perfect reconstruction formulas and bounds on aliasing error in sub-nyquist nonuniform sampling of multiband signals," IEEE Trans. Inform. Theory, vol. 46, no. 6, pp. 2173-2183, Sep. 2000.

[3] M. Mishali and Y. C. Eldar, "Blind multi-band signal reconstruction: Compressed sensing for analog signals," CCIT Report no. 639, EE Dept., Technion - Israel Institute of Technology; submitted to IEEE Trans. Signal Processing, Sep. 2007.

[4] M. Fleyer, A. Rosenthal, A. Linden, and M. Horowitz, "Multirate synchronous sampling of sparse multiband signals," Arxiv preprint arXiv:0806.0579, 2008.

[5] J. N. Laska, S. Kirolos, M. F. Duarte, T. S. Ragheb, R. G. Baraniuk, and Y. Massoud, "Theory and implementation of an analog-to-information converter using random demodulation," in Proc. of. ISCAS 2007, 2007, pp. 1959-1962.

[6] M. Mishali and Y. C. Eldar, "Reduce and boost: Recovering arbitrary sets of jointly sparse vectors," IEEE Trans. Signal Processing, vol. 56, no. 10, pp. 4692-4702, Oct. 2008.

[7] D. L. Donoho and M. Elad, "Maximal sparsity representation via $\ell 1$ minimization," Proc. Natl. Acad. Sci., vol. 100, pp. 2197-2202, Mar. 2003.

[8] J. Chen and X. Huo, "Theoretical results on sparse representations of multiple-measurement vectors," IEEE Trans. Signal Processing, vol. 54, no. 12, pp. 4634-4643, Dec. 2006.

[9] E. J. Candès, J. Romberg, and T. Tao, "Robust uncertainty principles: Exact signal reconstruction from highly incomplete frequency information," IEEE Trans. Inform. Theory, vol. 52, no. 2, pp. 489-509, Feb. 2006.

[10] D. L. Donoho, "Compressed sensing," IEEE Trans. Inform. Theory, vol. 52, no. 4, pp. 1289-1306, April 2006.

[11] S. F. Cotter, B. D. Rao, K. Engan, and K. Kreutz-Delgado, "Sparse solutions to linear inverse problems with multiple measurement vectors," IEEE Trans. Signal Processing, vol. 53, no. 7, pp. 2477-2488, July 2005.

[12] J. A. Tropp, A. C. Gilbert, and M. J. Strauss, "Algorithms for simultaneous sparse approximation. Part I: Greedy pursuit," Signal Process., vol. 86, pp. 572-588, Apr. 2006.

[13] J. A. Tropp, "Algorithms for simultaneous sparse approximation. Part II: Convex relaxation," Signal Process., vol. 86, pp. 589-602, Apr. 2006.

[14] M. Mishali, Y. C. Eldar, and J. A. Tropp, "Efficient sampling and stable reconstruction of wide band sparse analog signals," CCIT Report no. 705, EE Dept., Technion - Israel Institute of Technology, Oct. 2008.

[15] T. Tao and V. Vu, "On random \pm 1 matrices: Singularity and determinant," Random Structures Algorithms, vol. 28, no. 1, pp. 1-23, 2006.

[16] R. Baraniuk, M. Davenport, R. DeVore, and M. Wakin, "A simple proof of the restricted isometry property for random matrices," Const. Approx., 2007.

[17] T. Blumensath and M. E. Davies, "Sampling theorems for signals from the union of linear subspaces," preprint, 2007. 